\title{
PAPER
}

\section{Absorption characteristics of fibrous material covered with perforated facing and film}

\author{
Satoshi Sugie ${ }^{1, *}$, Junichi Yoshimura ${ }^{1}$ and Hiromasa Ogawa ${ }^{1}$ \\ ${ }^{1}$ Kobayasi Institute of Physical Research, \\ Higashi-motomachi 3-20-41, Kokubunji, 185-0022 Japan
}

(Received 4 April 2005, Accepted for publication 8 September 2005)

\begin{abstract}
We propose a method for predicting the absorption characteristics of a fibrous material, that is glass wool, covered with a perforated facing and an impermeable film, typically used for noise barriers. The method is based on Ingard and Bolt's model. It accounts for interactions among perforated facing, film and fibrous materials. The interaction occurs in areas where they are close to each other. That area was determined empirically as the coverage. The coverage is approximately $10 \mathrm{~mm}$ for a perforated facing with a 0.2 open area ratio. In the coverage, the perforated facing increases the acoustic impedance of film and fibrous material according to distance. The fibrous material causes acoustic resistance to the film when the film contacts the fibrous material. The formulae for their acoustic impedances were derived from many results of acoustic impedance measured using an impedance tube. The end correction of holes of the perforated facing was modified using the relationship between the measured values of resonance frequency for Helmholtz resonators with the perforated plate and their open area ratio. Results predicted by this method agree well with measured results obtained in most instances.
\end{abstract}

Keywords: Acoustic impedance, Absorption coefficient, Perforated facing, Film, Fibrous material

PACS number: 43.55.Ev [DOI: 10.1250/ast.27.87]

\section{INTRODUCTION}

Fibrous materials covered with a perforated facing and a film are commonly used in architectural acoustics and noise control situations. The perforated facing is used to protect the fibrous material from external forces. The impermeable film waterproofs the fibrous material and prevents fibers from being scattered. The perforated facing and film allow absorption reduction at high frequencies: the absorption peak shifts to a lower frequency. However, Davern [1] has reported that the absorption coefficient of the absorber decreases when an impermeable film is pinched between the perforated facing and the fibrous material. This phenomenon sometimes occurs in absorbers of noise barriers that are set under ceilings of double-deck viaducts.

Davern reported the measured results of many types of absorber consisting of a perforated facing, a film and a fibrous material. Nevertheless, the predictive formula for the absorber is not derived in that report. Since the study by Bolt, prediction for acoustic impedance of an absorb-

*e-mail: sugie@kobayasi-riken.or.jp ing material covered with a perforated facing has been studied in several works [2-7]. Numerous studies have also examined absorbent materials covered with impermeable films [8-10]. For predicting absorption the characteristics of fibrous materials combining both a perforated facing and a film, however, no investigations have been undertaken to date.

If the perforated facing, film, and fibrous material are mutually independent, the phenomenon cannot be explained theoretically. For that reason, it is necessary to consider their interaction to predict the absorption characteristics of this type of absorber.

In this study, we focus on a very thin perforated facing - approximately $1 \mathrm{~mm}$ thick — with a high open area ratio. Such facings are widely used in outdoor noise barriers for noise control. After measurement results for several types of the absorber are shown in this study, Ingard and Bolt's model is modified based on our results. Then, we propose a prediction method of acoustic impedance for a fibrous material covered with a perforated facing and a film, taking into account their mutual interaction. 


\section{INTERACTIONS AMONG PERFORATED FACING, FILM AND FIBROUS MATERIAL}

\subsection{Measurement Apparatus}

The impedance tube method was used for measuring the acoustic impedance and absorption coefficient of a fibrous material covered with facings. The impedance tube was made of acrylic resin and had 5-mm-thick walls and a $100 \mathrm{~mm}$ internal diameter. The normal surface impedance at the specimen surface was measured using a transferfunction method in which a single movable microphone is aligned with its axis at the center of the impedance tube (i.e., the one-microphone method) [11]. The frequency resolution is $6.25 \mathrm{~Hz}$. The upper working frequency of this tube was approximately $2,000 \mathrm{~Hz}$. Results with frequencies over $2,000 \mathrm{~Hz}$ are shown for reference in the figures. The measured acoustic impedance was obtained as a ratio to the characteristic impedance of air.

The impedance tube was used in the vertical position, as shown in Fig. 1, so that the conditions of measurement were unchanged in all measurement cases. The film was not bonded to the perforated facing and fibrous material; it was simply piled onto the fibrous material or the perforated facing.

\subsection{Perforated Facing and Fibrous Material}

Figure 2 shows the absorption coefficient of an absorber that consists of the fibrous material and the perforated facing, in comparison with an absorber having an air space $(5 \mathrm{~mm}$ thick) between the fibrous material and

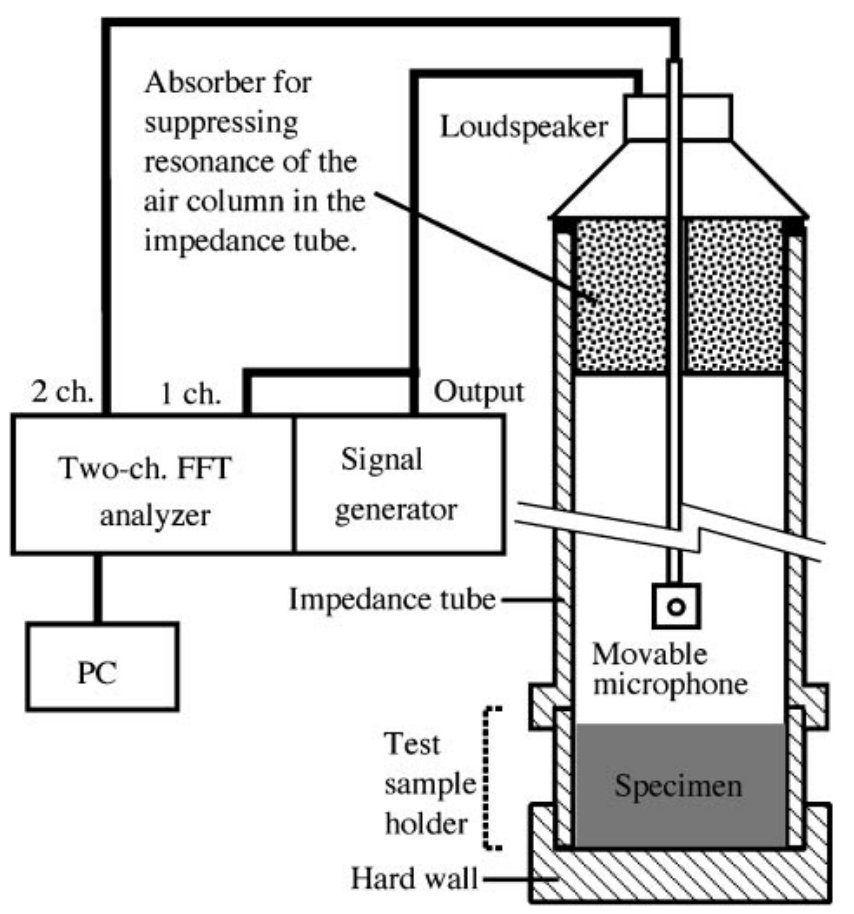

Fig. 1 Measurement apparatus.

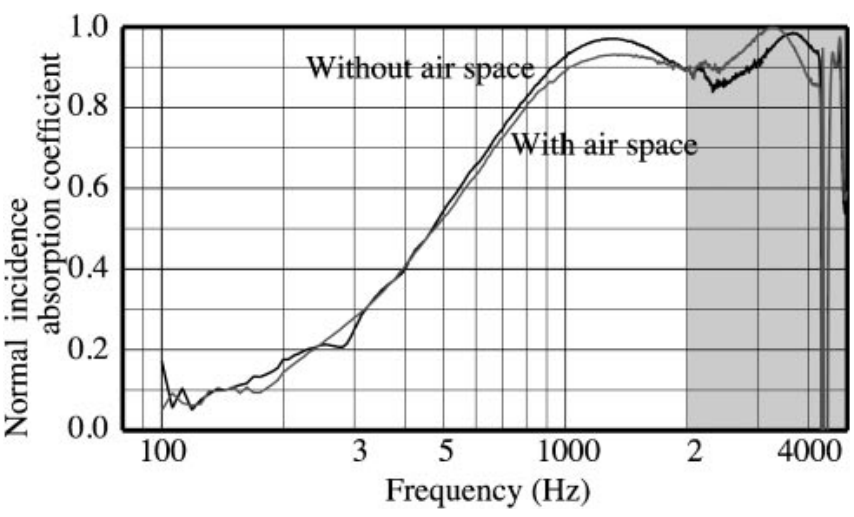

Fig. 2 Effect of air space between perforated facing and fibrous material; the perforated facing has $0.8 \mathrm{~mm}$ thickness, a 0.31 open area ratio, and a $10 \mathrm{~mm}$ hole diameter. The fibrous material is glass wool $(32 \mathrm{~kg} /$ $\mathrm{m}^{3}$ ): $50 \mathrm{~mm}$ thick and backed with a hard wall.

the perforated facing. The absorption coefficient of the absorption peak in the frequency region from 1,000 to $2,000 \mathrm{~Hz}$ for the absorber without the air space is greater than that with the air space. Comparison shows that the contact of perforated facing with fibrous material engenders higher absorption.

\subsection{Perforated Facing and Film}

Absorption coefficient measurements were made under the two conditions shown in Fig. 3. In Condition 1, the film is separate from the perforated facing. In Condition 2, the film is in contact with the perforated facing, but is not bonded to the perforated facing. The open area ratio of the perforated facing and the diameter of holes in the perforated facing were 0.19 and $10 \mathrm{~mm}$, respectively.

Measurement results are shown in Fig. 4, for comparison of both conditions without the perforated facing. Without the perforated facing, there is a slight difference between both conditions. The absorption peak frequency shifts slightly to lower frequencies. On other hand, although these results differs only with respect to the

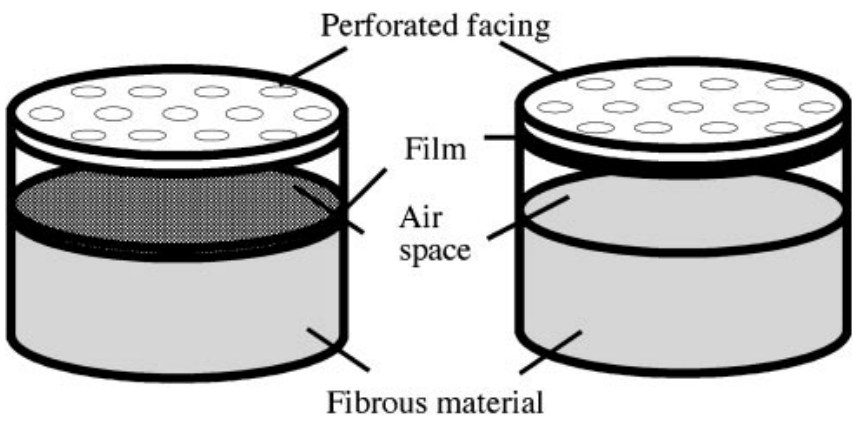

[Condition 1]

[Condition 2]

Fig. 3 Position of film between perforated facing and fibrous material. 


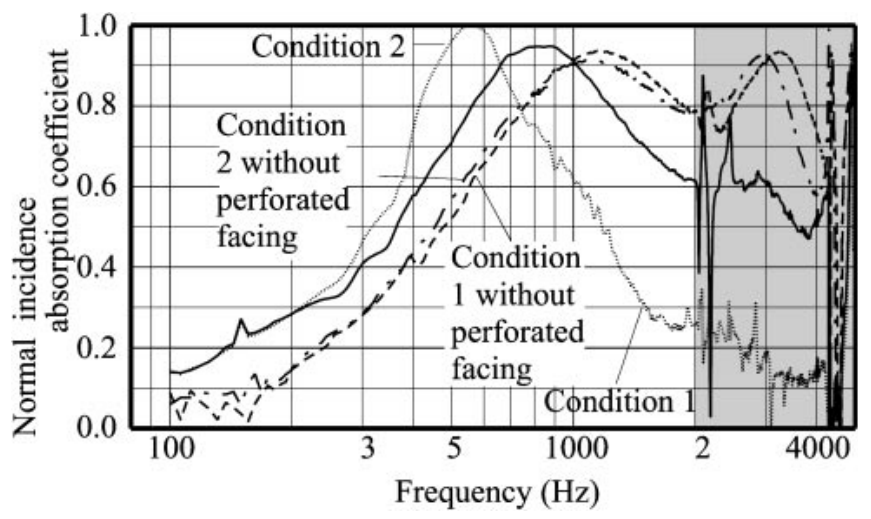

Fig. 4 Effect of film position between perforated facing and fibrous material: the perforated facing has $0.8 \mathrm{~mm}$ thickness, a 0.19 open area ratio, and a $10 \mathrm{~mm}$ hole diameter. The air space is $5 \mathrm{~mm}$ thick; the film has a $0.0268 \mathrm{~kg} / \mathrm{m}^{2}$ surface density. The material is glass wool $\left(32 \mathrm{~kg} / \mathrm{m}^{3}\right)$, which is $50 \mathrm{~mm}$ thick and backed with a hard wall.

film's position, the absorption peak for the film in contact with the perforated facing (Condition 2) appears at a lower frequency than that for the film separated from the facing (Condition 1). The absorption coefficient for Condition 2 decreases more than for Condition 1 at high frequencies. The absorption of the absorber with the perforated facing changes more widely than that of the absorber without the perforated facing. These results imply that the perforated facing determines film absorption characteristics.

\subsection{Film and Fibrous Material}

The acoustic internal resistance of the film is sufficiently smaller, as to be negligible, than that of the backing fibrous material because the film considered here is very thin. However, when the film touches the fibrous material, the resistance increases. For that reason, we measured the acoustic resistances of PVF films with various surface densities (various thicknesses), using the impedance tube method as shown in Fig. 5. The acoustic resistance $r_{\mathrm{f}}$ of a film is estimated by measuring the acoustic resistance $r_{2}$ on the surface of the film under two conditions - a film backed with an air layer and one backed with glass wool $\left(32 \mathrm{~kg} / \mathrm{m}^{3}\right)-$ as

$$
r_{\mathrm{f}}=r_{2}-r_{\mathrm{b}},
$$

where $r_{\mathrm{b}}$ is the acoustic resistance on the surface of the backing material. In the case where the backing material is air, $r_{\mathrm{b}}$ is obtained as $r_{\mathrm{b}}=\operatorname{Re}\left(z_{1}\right)$ and $z_{1}=\operatorname{coth}(i k l)$, where $k$ represents the wave number in air and $l$ is the thickness of the backing air layer. If the backing material was glass wool, $r_{\mathrm{b}}$ was measured.

The acoustic resistances of films backed with an air space (100 mm thickness) are shown in Fig. 6(a). The films were placed on a loose mesh to maintain the air space

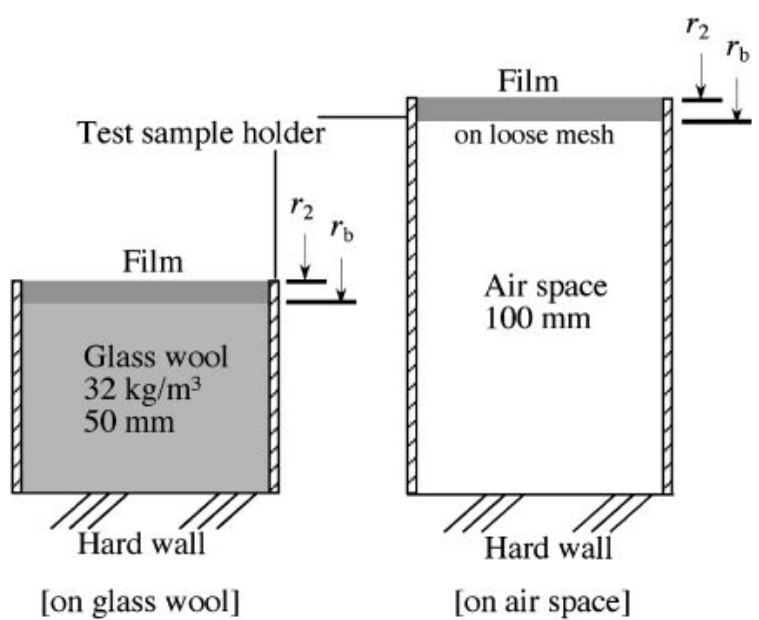

Fig. 5 Measurement of acoustic resistance of film using impedance tube.

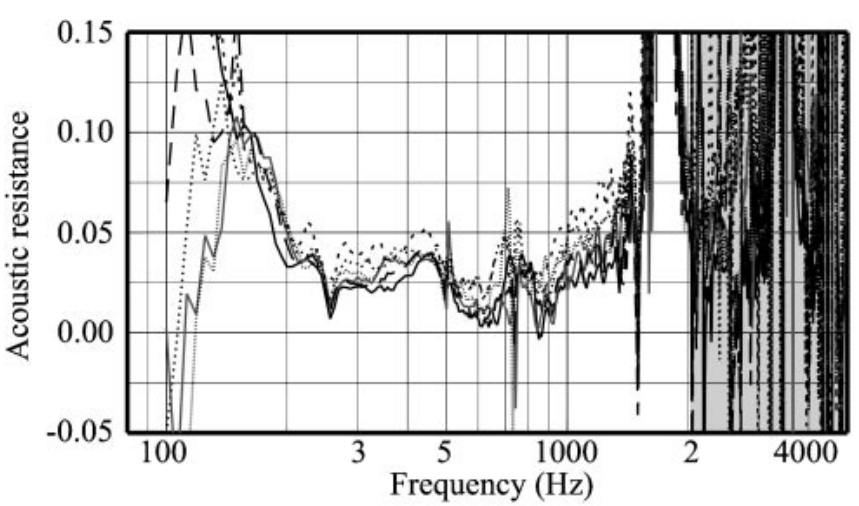

(a) on air space

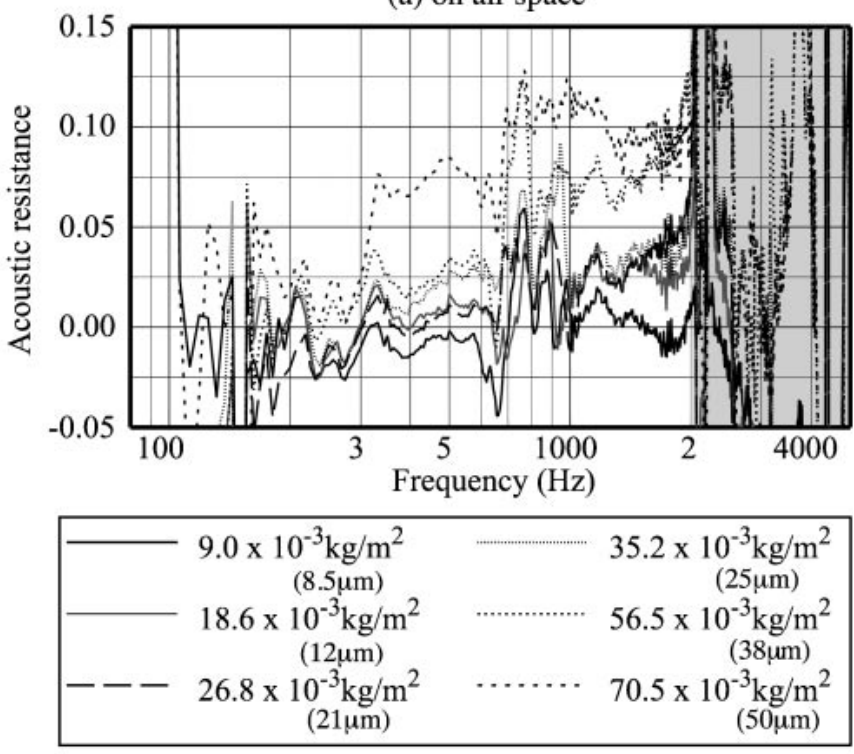

(b) on glass wool

Fig. 6 Comparison of acoustic resistances of films with various surface density. The surface densities are 9.0, $18.6,26.8,35.2,56.5$, and $70.5 \times 10^{-3} \mathrm{~kg} / \mathrm{m}^{2}$.

below the films. Replacing the air space with glass wool $\left(32 \mathrm{~kg} / \mathrm{m}^{3}\right)$ of $50 \mathrm{~mm}$ thickness, the acoustic resistances of films on the space are shown in Fig. 6(b). 
Figure 6(a) shows that films have a slight acoustic resistance that remains constant against increasing surface density. On the other hand, the acoustic resistance increased with increasing surface density when the film touched the fibrous material, as Fig. 6(b) shows. These phenomena also suggest that the fibrous material affects film absorption characteristics.

\section{EMPIRICAL FORMULAE FOR ACOUSTIC IMPEDANCE OF INDIVIDUAL MATERIALS}

\subsection{Perforated Facing}

According to Ingard [3], the effect of the perforated facing corresponds to an addition of mass reactance of air in each hole to the normal surface impedance under the perforated facing, whereas its acoustic resistance is negligible. Thus, assuming that the perforated facing has circular holes, the characteristic impedance $z_{\mathrm{p}}$ can be written as

$$
z_{\mathrm{p}}=i \frac{\omega}{c} \frac{\left(t_{\mathrm{p}}+\delta d\right)}{\phi},
$$

where $t_{\mathrm{p}}$ is the perforated facing thickness, $\delta$ is the end correction factor, $d$ is the hole diameter in the perforated facing, $\phi$ is the open area ratio of the perforated facing, $c$ is the sound velocity in the air, $\omega=2 \pi f$, and $f$ is the frequency.

Because the end correction factor $\delta$ depends not only on the diameter $d$ but also on the separation between holes, $\delta$ is also related to the open area ratio $\phi$. According to Byrne [5], $\delta$ is obtained as

$$
\delta=0.48(\sqrt{\pi}-2.5 \sqrt{\phi}) .
$$

However, this equation shows that $\delta$ is a negative value when $\phi>\pi / 2.5^{2}=0.5$. In this study, we assume that the perforated facing is very thin and has a high open area ratio. Consequently, this equation is unsuitable for predicting the absorption characteristics of a perforated facing in this case.

For that reason, the end correction factor $\delta$ was estimated experimentally. The acoustic reactances of several types of perforated facing $(0.8 \mathrm{~mm}$ thickness $)$ were measured as having an air space $(50 \mathrm{~mm}$ thick) behind them. Equation (4) resulted from the equation for calculating resonance frequency of air space with perforated facing. Figure 7 shows the relationship between the obtained end correction factor $\delta$ by inserting the resonance frequency $f_{0}$ into Eq. (4) and the open area ratio of the perforated facing. The resonance frequency $f_{0}$ was calculated as though the reactance was equal to zero.

$$
\delta=\left(\frac{\phi}{2 \pi / c f_{0} \tan \left(2 \pi / c f_{0} h\right)}-t_{\mathrm{p}}\right) / d
$$

In that equation, $\phi=\pi(d / 2)^{2} n / 0.05^{2} \pi, h$ is the thickness of air space and $n$ is the number of holes in a sheet with

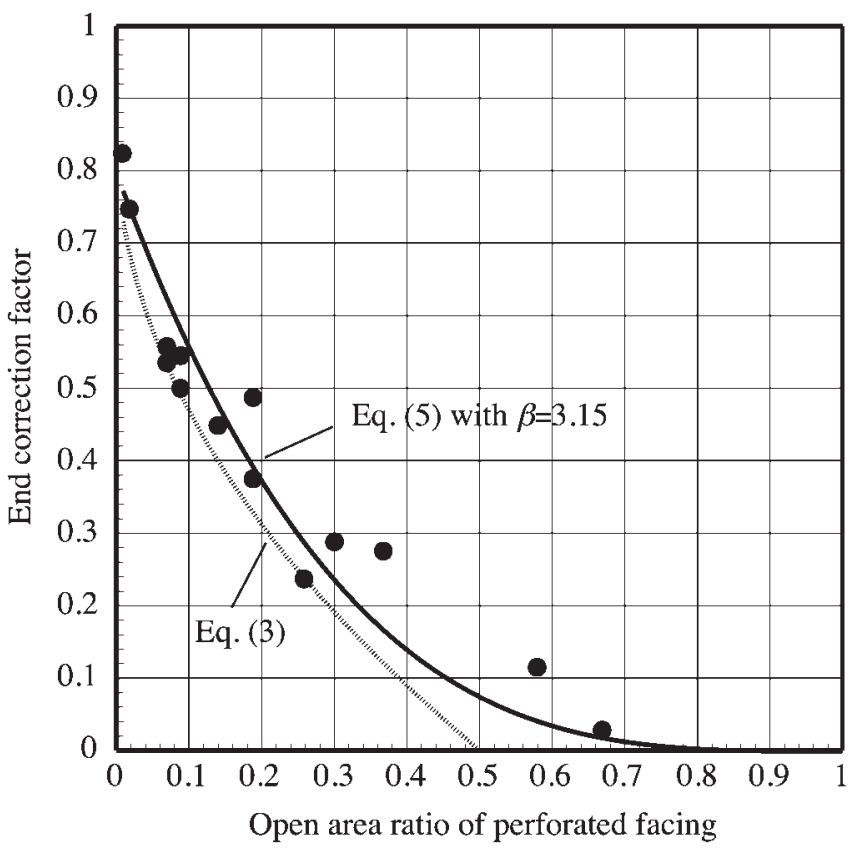

Fig. 7 Relationship between open area ratio and endcorrection factor of perforated facing. Filled circles: measurement results. Dotted line: results estimated using Eq. (3). Solid line: results estimated using Eq. (5) with $\beta=3.15$.

$100 \mathrm{~mm}$ diameter of the perforated facing, used as a specimen. The estimated values of end correction factors given by Eq. (3) are also shown in Fig. 7. They are smaller than the measured results, which indicate that the end correction factor $\delta$ depends on the open area ratio; it does not become zero if the open area ratio is greater than 0.5 . Therefore, $\delta(\phi)$ can be expressed in terms of the open area ratio $\phi$ based on the measurement results. It must satisfy

$$
\begin{aligned}
& \lim _{\phi \rightarrow 0} \delta(\phi)=0.8, \\
& \delta(1)=0, \\
& \left.\frac{d}{d \phi} \delta(\phi)\right|_{\phi=1}=0 .
\end{aligned}
$$

Accordingly, $\delta(\phi)$ is given as

$$
\delta(\phi)=0.8(1-\phi)^{\beta},
$$

where the estimated value of $\beta$ is 3.15 (based on leastsquares fitting of the results shown in Fig. 7).

\subsection{Film under Perforated Facing}

According to Kuttruff [12], the film effect is described by the addition of the mass reactance of the film to the normal surface impedance under the film. However, the results shown in Fig. 4 indicate that the absorption characteristics of a film in contact with the perforated facing are determined more by the perforated facing than by other factors. Therefore, the results cannot be explained 


\section{Perforated facing}

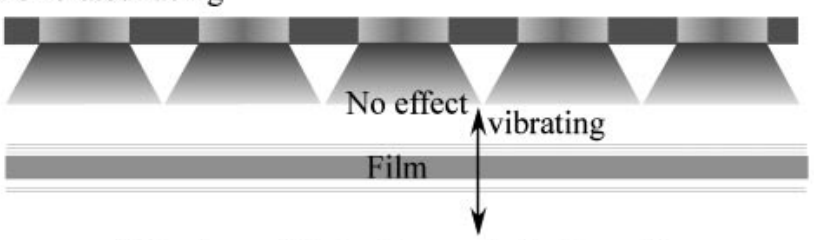

(a) Perforated facing is enough far from film.

Perforated facing

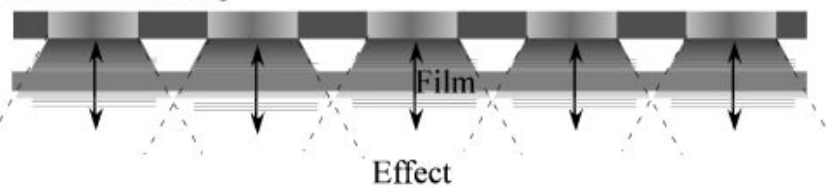

(b) Perforated facing is close to film

Fig. 8 Model of behavior of film under perforated facing.

by the addition of only the mass reactance of the film.

The perforated facing affects the film because it controls the film vibration when they are placed close to each other. Only the film in the area under each hole of the perforated facing is assumed to vibrate; therefore, the size of this area would increase with increasing distance between the perforated facing and film (Fig. 8). Consequently, the characteristic impedance of film $z_{\mathrm{f}}$ is obtainable as

$$
z_{\mathrm{f}}=\left(r_{\mathrm{f}}+i \frac{1}{\rho c} m_{\mathrm{f}} \omega\right) / \psi(x),
$$

where $r_{\mathrm{f}}$ represents the acoustic resistance of the film on the fibrous material, $m_{\mathrm{f}}$ is the film surface density, $\psi(x)$ is a function of the distance $x$ between the perforated facing and the film, and $\rho$ is the air density.

When $x$ is larger than the distance $a, \psi(x)$ is equal to one; at that distance, the effect becomes negligible. The simplest approximation is to assume that $\psi(x)$ varies linearly with the open area ratio of perforated facing. That approximation can be written as

$$
\begin{aligned}
& \psi(x)=\phi+\frac{x}{a}(1-\phi), \quad(x<a), \\
& \psi(x)=1, \quad(x \geq a) .
\end{aligned}
$$

Distance $a$ is defined as the coverage hereafter. It is estimated experimentally. Specimens consisted of a perforated facing ( 0.19 open area ratio, $0.8 \mathrm{~mm}$ thickness and $10 \mathrm{~mm}$ hole diameter), an air space, a film, and a fibrous material (glass wool $32 \mathrm{~kg} / \mathrm{m}^{3}, 50 \mathrm{~mm}$ ) under "Condition 1" in Fig. 3. Measurement results of the absorption coefficient and acoustic reactance were obtained for a number of air spaces with different parameters. Those results are shown in Fig. 9. The resonance frequency, at which the acoustic reactance crosses the zero line, appears from $500 \mathrm{~Hz}$ to $1,000 \mathrm{~Hz}$. Resonance frequency shifts to
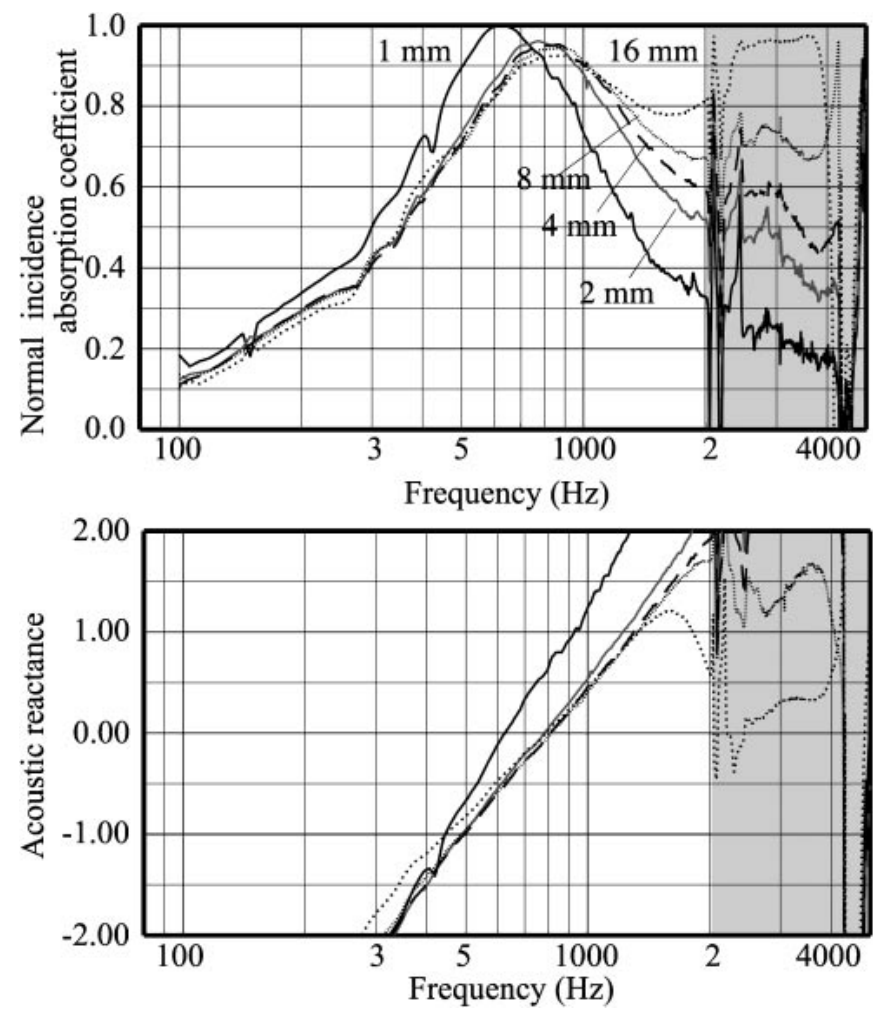

Fig. 9 Effect of distance between film and perforated facing under Condition 1: the distances are 1, 2, 4, 8, and $16 \mathrm{~mm}$; other parameters are identical to those in Fig. 4.

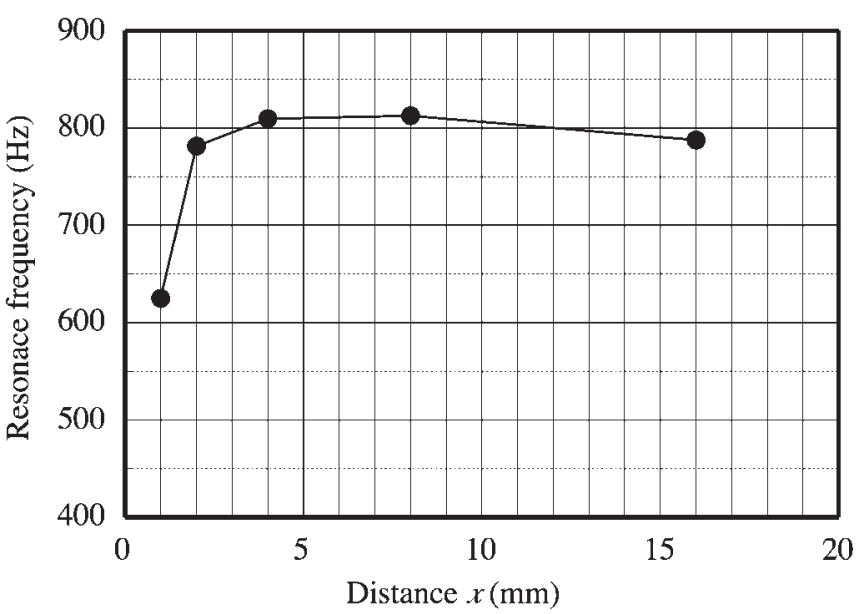

Fig. 10 Relationship between resonance frequency and distance $x$ between perforated facing and film.

higher values as the air space thickness increases.

The relationship between resonance frequency and distance $x$ is, shown in Fig. 10. Resonance frequency increased sharply as the separation between the perforated facing and the film was increased from 1 to $2 \mathrm{~mm}$. Separations from 2 to $8 \mathrm{~mm}$, however, produced little change; separations above $8 \mathrm{~mm}$ produced a slight decrease in resonance frequency. On the other hand, the resonance 
frequency must decrease gently with increasing distance in the case that the perforated facing does not affect film vibration, because the total thickness of the perforated facing, air space, film and fibrous material increases [13]. The decrease in the acoustic reactance of the film increases resonance frequency as separation increases from $0 \mathrm{~mm}$. The increase in the total thickness of the absorber decreases resonance frequency when the separation is above the coverage $a$. Therefore, it may be stated that the coverage $a$ is approximately $5-10 \mathrm{~mm}$ because the results of the resonance frequency are stable or decrease gradually in the distance range in Fig. 10. Ingard proposed that the distance corresponded to the holes' diameter [4]. Therefore, we infer that the result obtained herein coincides with those obtained by Ingard.

The coverage $a$ must depend on the hole diameter of the perforated facing. If its open area ratio is close to unity, the coverage $a$ must be close to zero. Therefore, the coverage $a$ must also depend on the open area ratio of perforated facing. The end correction factor $\delta$ is related to the open area ratio according to Eq. (5). If it is assumed that the coverage $a$ is obtained as

$$
a=2 \delta(\phi) d,
$$

substitution of Eq. (5), $10 \mathrm{~mm}$ diameter, and 0.19 open area ratio in this equation yields $a=8 \mathrm{~mm}$, which is nearly equal to the coverage obtained from the measurement result.

\subsection{Film on Fibrous Material}

In section 2.4, we explained that the acoustic resistance of a film is related to its surface density when the film is on a fibrous material. Figure 11 shows the relationship between the surface density and the arithmetic mean of the acoustic resistance of films in the frequency region from $500 \mathrm{~Hz}$ to $2,000 \mathrm{~Hz}$, which is shown in Fig. 6(b). The acoustic resistance is proportional to the surface density of the film. For that reason, we fitted a straight line to the results and obtained the approximation

$$
r_{\mathrm{f}}=1.5 m_{\mathrm{f}}-0.01 \text {. }
$$

\subsection{Fibrous Material under Perforated Facing}

The absorption coefficient for a fibrous material in contact with a perforated facing is greater than that for a fibrous material separated from a perforated facing in the frequency region from 1,000 to 2,000 Hz, as Fig. 2 shows. According to Ingard [3], acoustic resistance of the absorber is increased to $\sigma_{\mathrm{c}} l / \phi\left(\sigma_{\mathrm{c}}\right.$ : the airflow resistivity of absorber), when the perforated facing is in contact with a very thin absorber. Presumably, this phenomenon results from the change in the acoustic resistance of the fibrous material in coverage $a$. That is, when a certain part (with thickness $l^{\prime}$ ) of the fibrous material layer is put in coverage

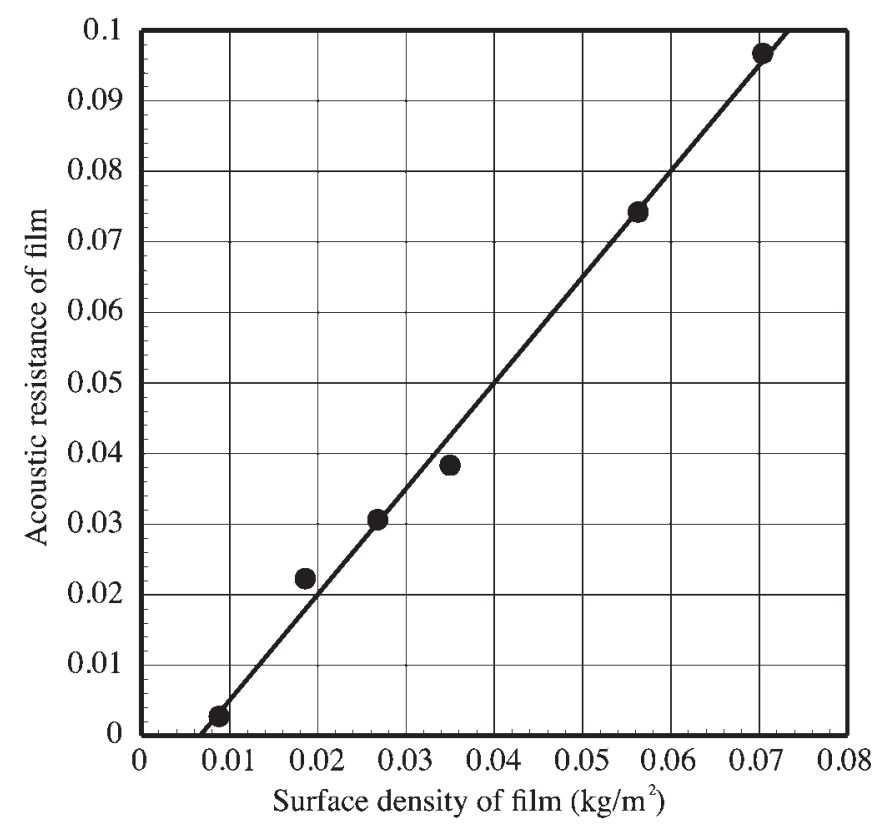

Fig. 11 Relationship between surface density and acoustic resistance of film.

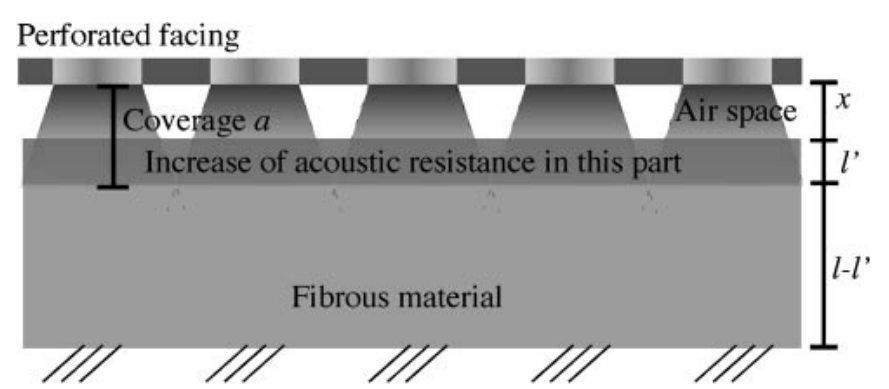

Fig. 12 Concept of perforated facing's effect on fibrous material in case that $x$ is smaller than coverage $a$.

$a$, as shown in Fig. 12, the acoustic resistance of the part increases, but that of the fibrous material layer behind the part remains unchanged. Therefore, the normal surface impedance $z_{1}$ on the surface of the fibrous material is

$z_{1}=\frac{1}{\psi(x)} \frac{\sigma_{\mathrm{c}} l^{\prime}}{\rho c}+z_{\mathrm{c}} \frac{z_{\mathrm{b}} \cosh \left\{\gamma_{\mathrm{c}}\left(l-l^{\prime}\right)\right\}+z_{\mathrm{c}} \sinh \left\{\gamma_{\mathrm{c}}\left(l-l^{\prime}\right)\right\}}{z_{\mathrm{c}} \cosh \left\{\gamma_{\mathrm{c}}\left(l-l^{\prime}\right)\right\}+z_{\mathrm{b}} \sinh \left\{\gamma_{\mathrm{c}}\left(l-l^{\prime}\right)\right\}}$,

$$
\begin{array}{ll}
l^{\prime}=a-x, & (x<a), \\
l^{\prime}=0, & (x \geq a),
\end{array}
$$

where $x$ is the distance between the perforated facing and the fibrous material, $\sigma_{\mathrm{c}}$ is the airflow resistivity of the fibrous material, and $z_{\mathrm{c}}, \gamma_{\mathrm{c}}$ and $l$ are the characteristic impedance, propagation constant and thickness of the fibrous material, respectively, and $z_{\mathrm{b}}$ is the normal acoustic impedance under the fibrous material. If $z_{\mathrm{b}}$ is infinite, that is, if a hard wall backs the fibrous material, $z_{1}$ is represented as 


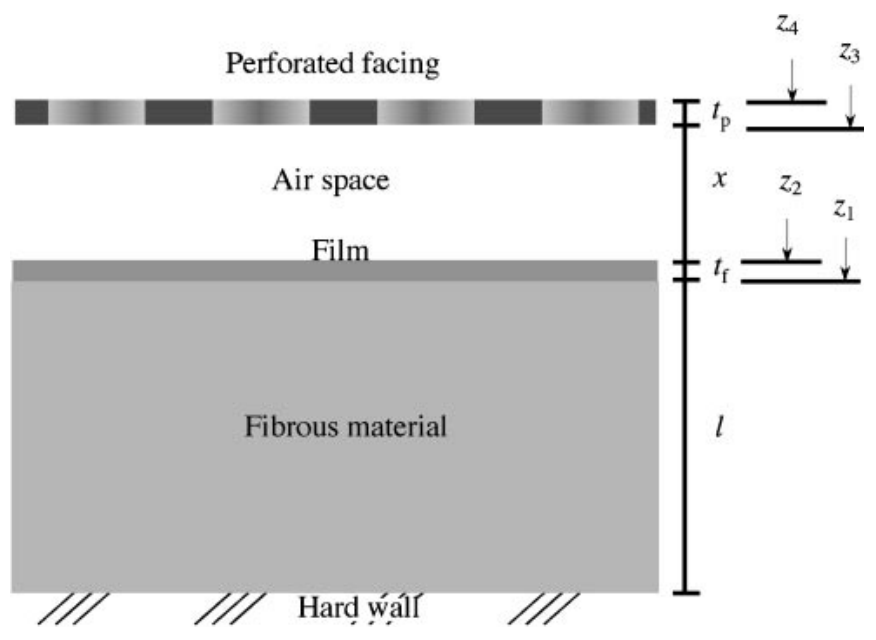

Fig. 13 Arrangement of absorber for prediction.

$$
z_{1}=\frac{1}{\psi(x)} \frac{\sigma_{\mathrm{c}} l^{\prime}}{\rho c}+z_{\mathrm{c}} \operatorname{coth}\left\{\gamma_{\mathrm{c}}\left(l-l^{\prime}\right)\right\}
$$

\section{DISCUSSION}

\subsection{Prediction Method}

The arrangement of the absorber is shown in Fig. 13 to predict the normal surface impedance ( $\rho c$ unit) and normal incidence absorption coefficient of the absorber. Estimating the characteristic impedance $z_{\mathrm{c}}$ and propagation constant $\gamma_{\mathrm{c}}$ of the fibrous material using the laws of Delany and Bazley modified by Miki [14], the normal surface impedance $z_{1}$ is obtained from Eq. (11). The normal surface impedance $z_{2}$ on a layer of the film is obtainable as

$$
z_{2}=z_{\mathrm{f}}+z_{1},
$$

where the characteristic impedance $z_{\mathrm{f}}$ of the film is calculated using Eqs. (6) and (9). The normal surface impedance $z_{3}$ for the layer of air space above the film is written as

$$
z_{3}=\frac{z_{2} \cosh (i k x)+\sinh (i k x)}{\cosh (i k x)+z_{2} \sinh (i k x)},
$$

where $k$ is the wave number in air. Thereby, the normal surface impedance $z_{4}$ on the perforated facing can be estimated as

$$
z_{4}=z_{\mathrm{p}}+z_{3},
$$

where the characteristic impedance $z_{\mathrm{p}}$ of the perforated facing is calculated using Eqs. (2) and (5). The normal incidence absorption coefficient $\alpha$ can be calculated as

$$
\alpha=1-\left|\frac{1-z_{4}}{1+z_{4}}\right|^{2} .
$$

\subsection{Prediction for Absorption Coefficient}

In this section, we present the predicted results of a normal incidence absorption coefficient for several types of

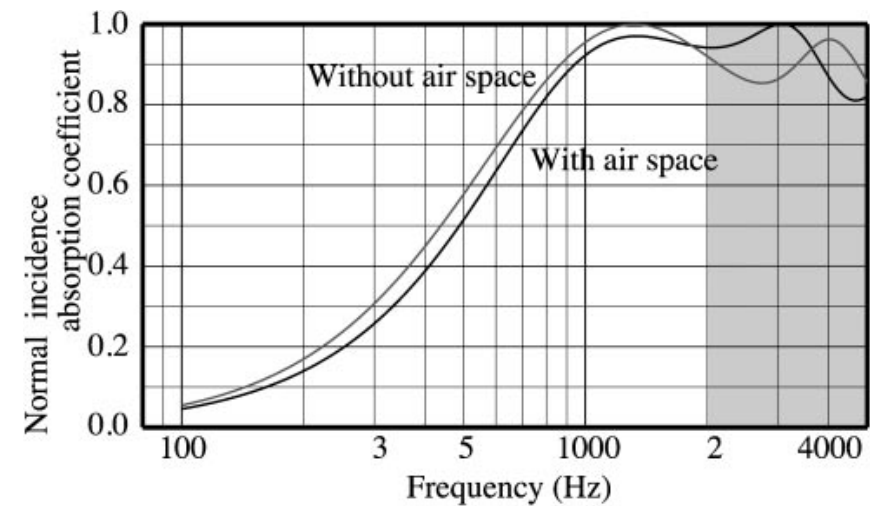

Fig. 14 Effect of air space between perforated facing and fibrous material for fibrous material covered with perforated facing, which has $0.8 \mathrm{~mm}$ thickness, 0.31 open area ratio, and $10 \mathrm{~mm}$ hole diameter (see Fig. 2).

absorber. The parameters for prediction are as follows. The fibrous material backed with a hard wall has a 10,000 $\mathrm{Pas} / \mathrm{m}^{2}$ airflow resistivity and a $50 \mathrm{~mm}$ thickness; the film has a $0.03 \mathrm{~kg} / \mathrm{m}^{2}$ surface density. Figure 14 shows a comparison of the calculated results for an absorber having an air space ( $5 \mathrm{~mm}$ thickness) between the perforated facing and the fibrous material with those for an absorber having no air space. No film separates the perforated facing and fibrous material in these cases. The absorption coefficient in the absorption peak in the frequency region from 1,000 to $2,000 \mathrm{~Hz}$ for the absorber having no air space was higher than in the absorber with air space, as the measured results in Fig. 2 show.

Figure 15 shows the calculated results for the absorption coefficient for the absorber under the two conditions shown in Fig. 3. The absorption peak under Condition 2 appeared at a lower frequency than that under Condition 1.

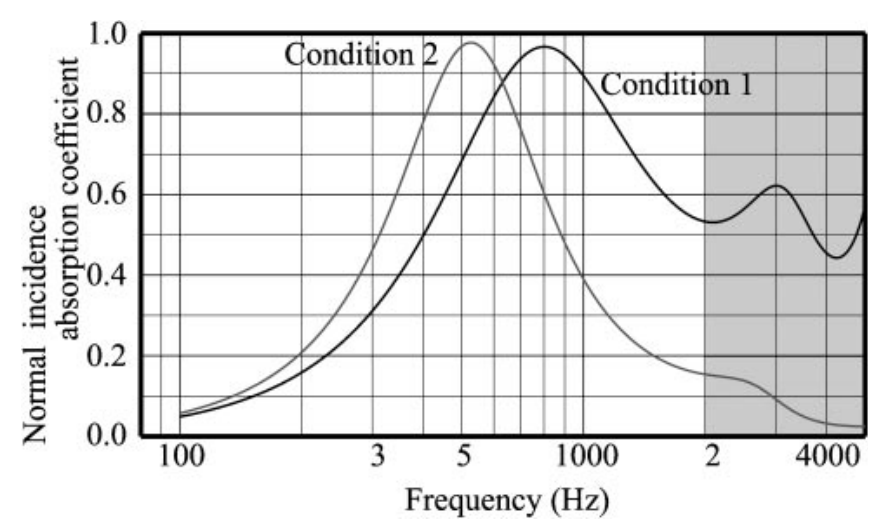

Fig. 15 Effect of film position between perforated facing and fibrous material for fibrous material covered with perforated facing. The perforated facing has a $0.8 \mathrm{~mm}$ thickness, a 0.2 open area ratio, and a $10 \mathrm{~mm}$ hole diameter. The distance between the perforated facing and fibrous material is $50 \mathrm{~mm}$ (see Fig. 4). 


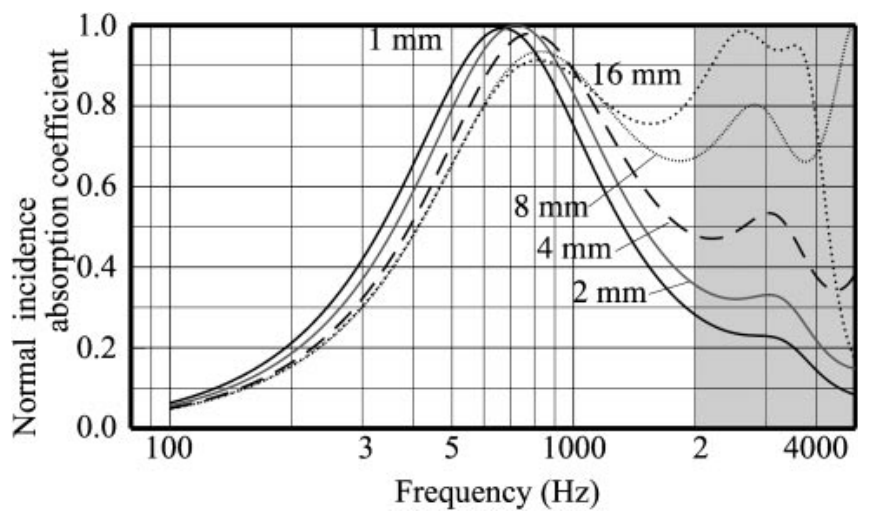

Fig. 16 Effect of air space between perforated facing and film for fibrous material covered with the perforated facing and film. The perforated facing has a $0.8 \mathrm{~mm}$ thickness, a 0.2 open area ratio, and a $10 \mathrm{~mm}$ hole diameter. The air space thicknesses are 1, 2, 4, 8, and $16 \mathrm{~mm}$ (see Fig. 9).

The predicted results for Condition 2 are smaller than for Condition 1 at high frequencies. Consequently, the calculated results correspond to the measured results, as shown in Fig. 4.

Figure 16 shows calculated results of the normal incidence absorption coefficient for absorbers with various air space thickness between the perforated facing and the film. The peak frequency of the absorption coefficient increases with greater thickness. The absorption coefficient in the high-frequency region increases concomitant with greater air-space thickness. These results are similar to the measurement results that are shown in Fig. 9.

\subsection{Comparisons between Predicted and Measured Results}

The measured and predicted values are compared using measured parameters. The airflow resistivity of fibrous material is $7,500 \mathrm{Pas} / \mathrm{m}^{2}$. This is estimated because the predicted values agree with the measured values for the case in which the fibrous material, whose thickness is $50 \mathrm{~mm}$, is backed with a hard wall.

Figure 17 shows a comparison of the measured and predicted values for the case in which $x$ is nearly zero. The predicted values were calculated with the distance $x$ between the perforated facing and film as $0 \mathrm{~mm}$. On the other hand, the measured results were represented for two cases: very thin air space $(2 \mathrm{~mm})$ and no air space. A slight difference exists in these two cases, but the absorption is very different.

The predicted values for the acoustic reactance concur well with the measured results (gray solid line) when $x$ is $2 \mathrm{~mm}$, but the measured acoustic resistance is higher than predicted. Accordingly, the absorption coefficients agree well with each other at the peak frequencies of the
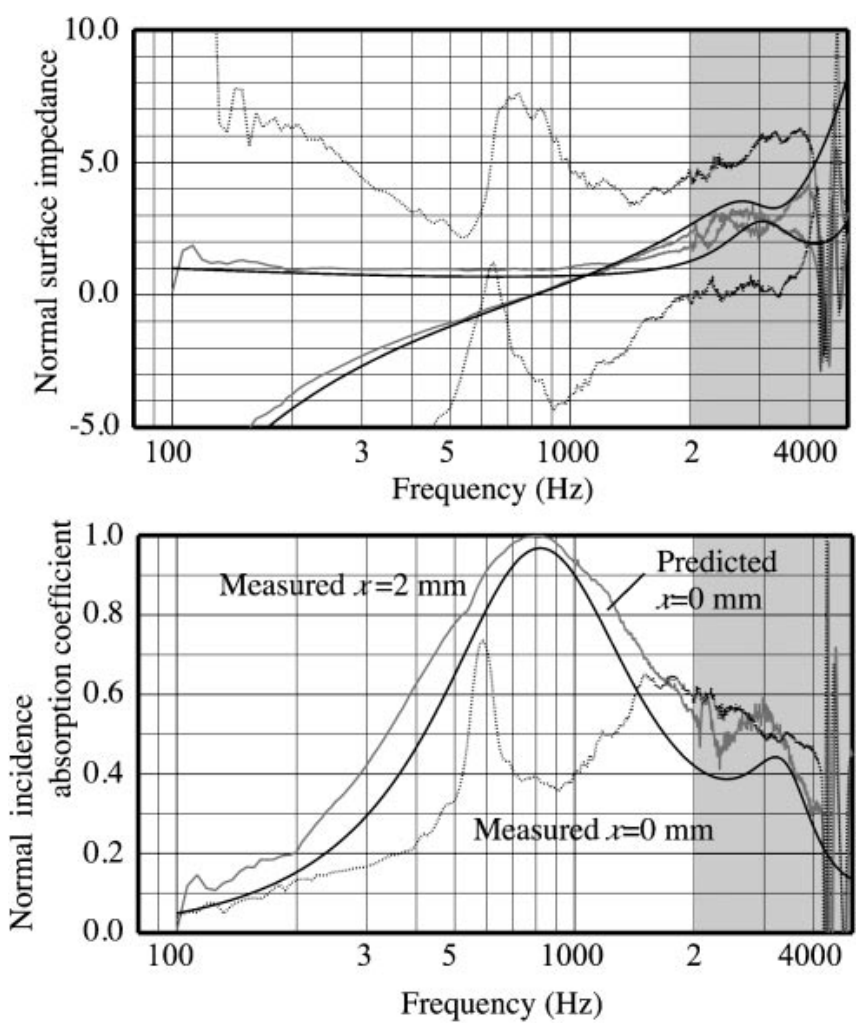

Fig. 17 Comparisons of measured and predicted values for fibrous material covered with perforated facing and film in case of $x \cong 0 ; m_{\mathrm{f}}=0.0268 \mathrm{~kg} / \mathrm{m}^{2}, \phi=0.31$, $t_{\mathrm{p}}=0.8 \mathrm{~mm}$, and $d=10 \mathrm{~mm}$.

absorption coefficient, but the magnitudes of the measured values are larger than those predicted.

When $x$ was $0 \mathrm{~mm}$ for the measured results, i.e., when the film is completely in contact with the perforated facing and fibrous material, the measured values (dotted line) do not agree with the predicted values at all. The absorption peak shows a sharper form at lower frequencies than that of the predicted absorption peak. The absorption coefficient decreased with frequencies not higher than $1,700 \mathrm{~Hz}$, whereas the acoustic resistances increased abruptly.

Disagreement between the measured and predicted values may result from other effects than those considered herein. In this case, the film is pinched between the perforated facing and the fibrous material. This pinching increases film stiffness. Therefore, an increase in acoustic resistance occurs and the absorption coefficient is reduced at high frequencies. For that reason, it is necessary to deduce another model for predicting the film's acoustic impedance in such cases.

The acoustic resistance must be related to the film's vibration velocity if the friction between the film and fibrous material increases the film's acoustic resistance. Even if the perforated facing almost touches the film, the vibration velocity of the film near the holes becomes extremely high. Therefore, an increase in the film's 

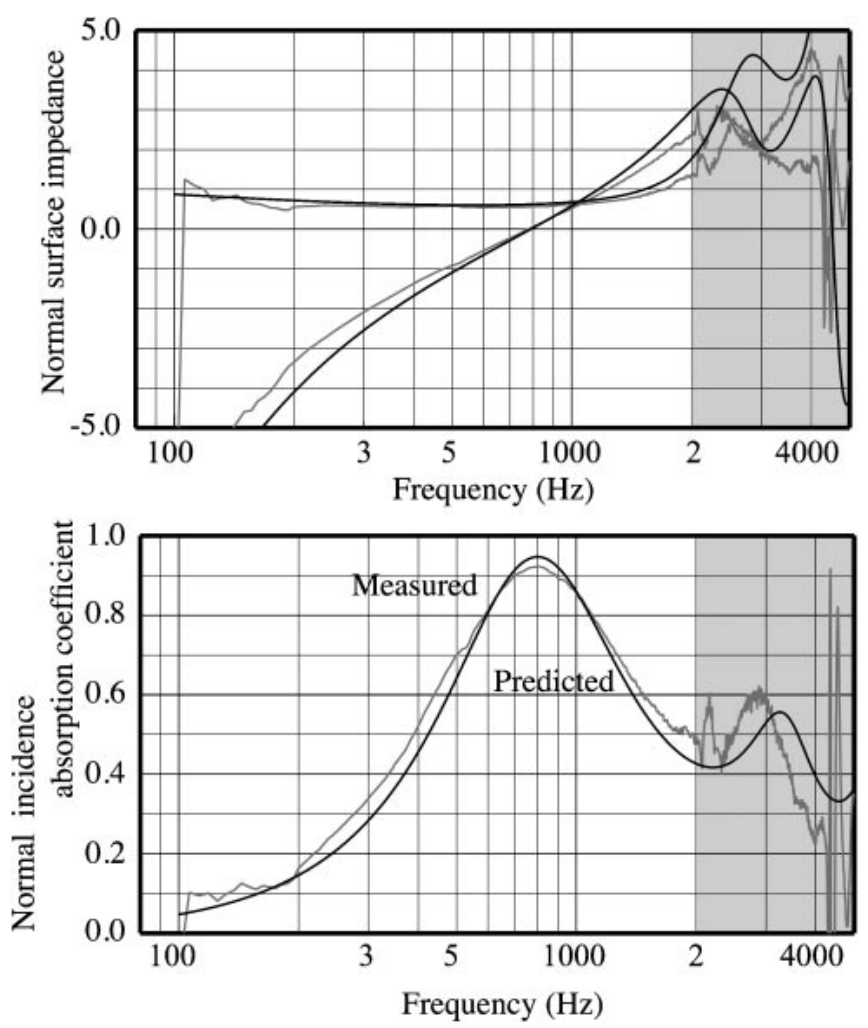

Fig. 18 Comparisons of measured and predicted values for fibrous material covered with perforated facing and film in case of $x \cong a / 2 ; m_{\mathrm{f}}=0.0268 \mathrm{~kg} / \mathrm{m}^{2}, x=4$ $\mathrm{mm}, \phi=0.19, t_{\mathrm{p}}=0.8 \mathrm{~mm}$, and $d=10 \mathrm{~mm}$.

resistance might result from the film's increased vibration velocity. Slight contact with the perforated facing might engender increased film resistance.

Figure 18 shows a comparison between the measured and predicted values in the case of $x \cong a / 2$. The distance $x$ was $4 \mathrm{~mm}$ and the coverage $a$ was estimated at $8 \mathrm{~mm}$ using Eq. (8) because the perforated facing has an open area ratio of 0.19 and $10 \mathrm{~mm}$ diameter holes. The predicted values agree with the measured values in this case.

Figure 19 shows a comparison between the predicted and measured values in the case of $x \cong a$. Because the perforated facing had an open area ratio of 0.37 and 10mm-diameter holes, $a$ was estimated at $4 \mathrm{~mm}$, which was equal to $x$. Despite a slight disagreement between the predicted and measured values in terms of the absorption peak, they approximately agreed. Figure 20 shows a comparison of the measured and predicted values when the perforated facing was separated from the film so that it does not affect the film $(x>a)$. The predicted values agreed well with the measured values.

We conclude that $a$ obtained by Eq. (8) is suitable for the coverage affected by the perforated facing. Figure 21 shows the relationship between $a$ and the open area ratio of the perforated facing obtained using Eq. (8) in comparison with that obtained as $a$ corresponds to the diameter $d$ of
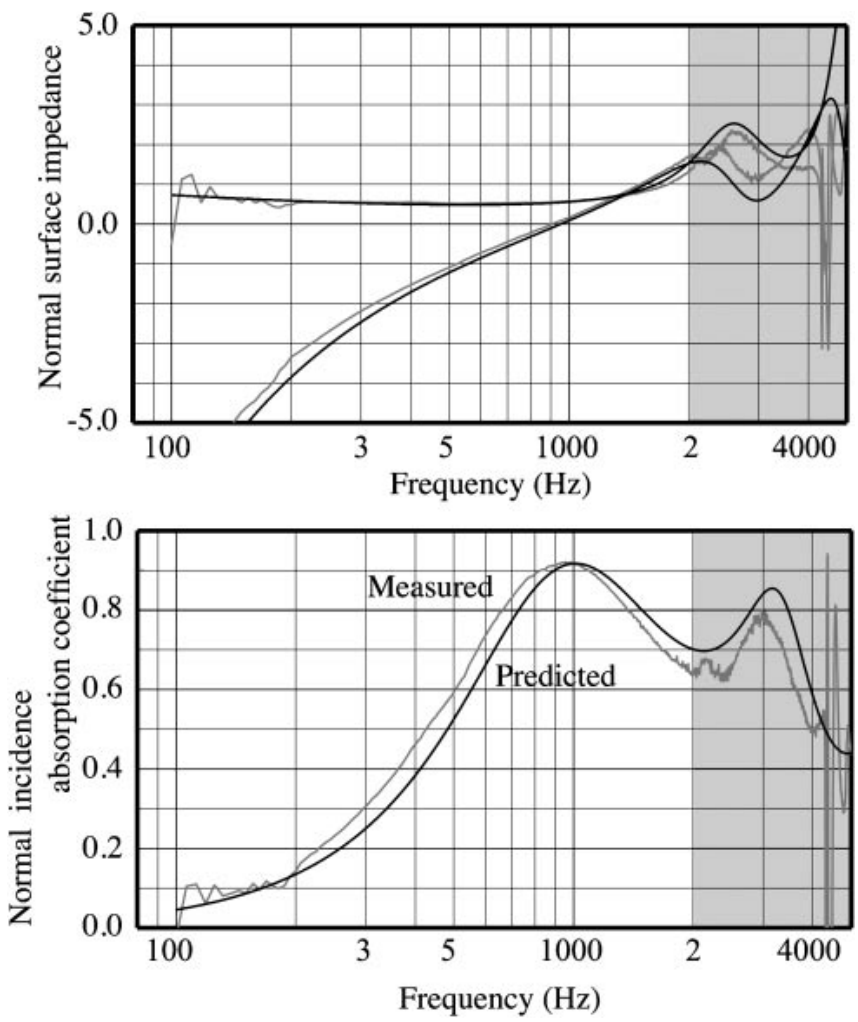

Fig. 19 Comparisons of measured and predicted values for fibrous material covered with perforated facing and film in case of $x \cong a ; m_{\mathrm{f}}=0.0268 \mathrm{~kg} / \mathrm{m}^{2}, x=4 \mathrm{~mm}$, $\phi=0.37, t_{\mathrm{p}}=0.8 \mathrm{~mm}$, and $d=10 \mathrm{~mm}$.
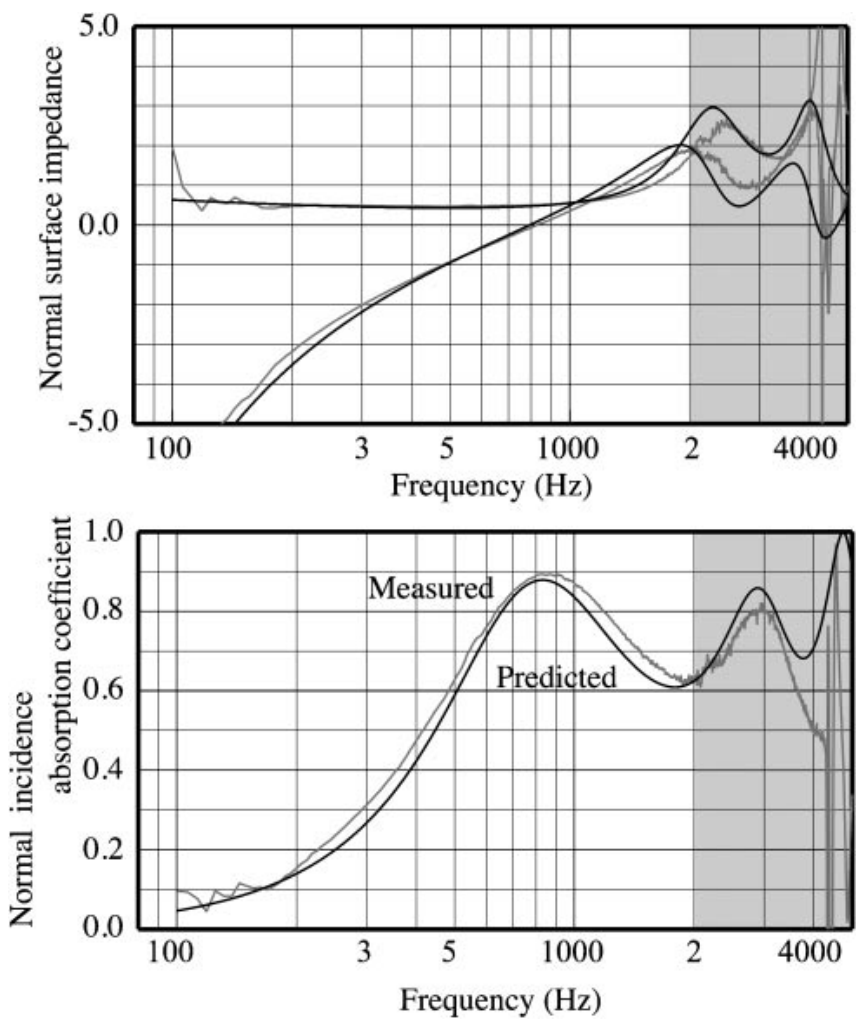

Fig. 20 Comparisons of measured and predicted values for fibrous material covered with perforated facing and film in case of $x>a ; m_{\mathrm{f}}=0.0268 \mathrm{~kg} / \mathrm{m}^{2}, x=9 \mathrm{~mm}$, $\phi=0.19, t_{\mathrm{p}}=0.8 \mathrm{~mm}$, and $d=10 \mathrm{~mm}$. 


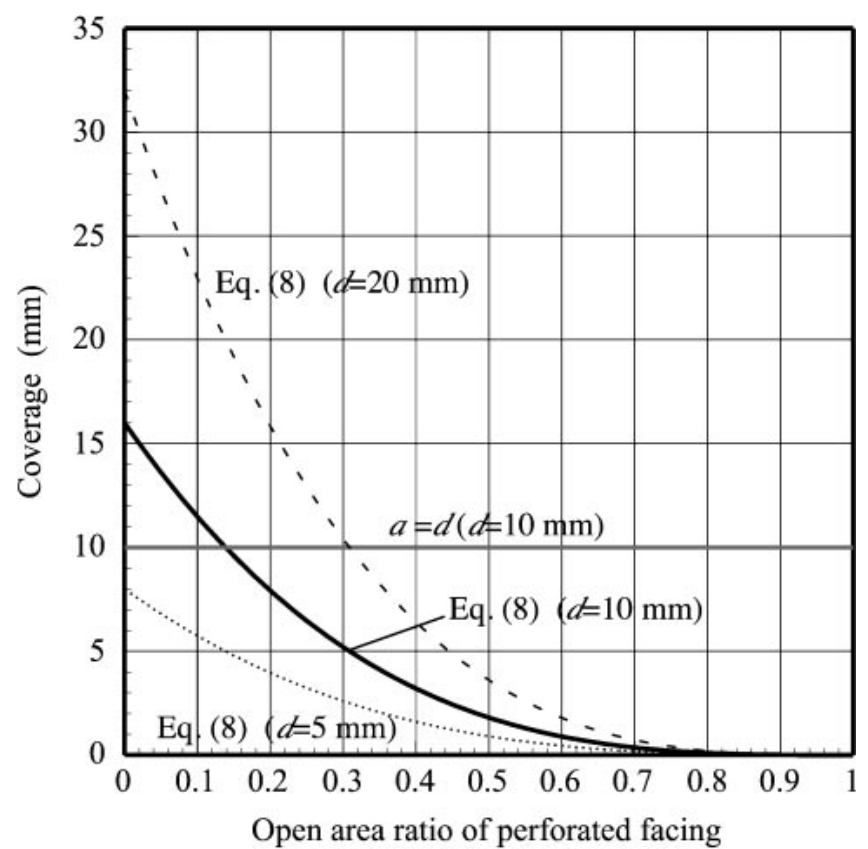

Fig. 21 Relationship between coverage $a$ and open area ratio of perforated facing.

holes. In the former relationship (black line) $a$ decreases as open area ratio increases. It becomes negligible at an open area ratio higher than 0.7 regardless of the diameter of holes, whereas in the latter relationship (gray line) $a$ remains constant with respect to open area ratio.

\section{CONCLUSIONS}

We have proposed a method for predicting the normal surface impedance and normal incidence absorption coefficient of a fibrous material, that is glass wool, covered with a thin perforated facing and an impermeable film. This method takes into account the following interactions.

(1) The perforated facing's control of the film vibration $\left(z_{\mathrm{f}} \propto 1 / \psi(x)\right)$.

(2) The fibrous material's increasing acoustic resistance of the film $\left(r_{\mathrm{f}}=1.5 m_{\mathrm{f}}-0.01\right)$.

(3) The perforated facing's addition of acoustic resistance to the acoustic impedance of the fibrous material, when in coverage $a$.

The results showed that coverage $a$ can be calculated as $a=2 \delta(\phi) d$. It was also considered that the end correction of the perforated facing depends on the open area ratio of the perforated facing. The relationship between them was obtained as $\delta(\phi)=0.8(1-\phi)^{3.15}$, measuring the resonance frequency of many resonators. Predictions and measurements agreed well regarding the normal surface impedance and the normal incidence absorption coefficient, except in cases where the film was pinched by the perforated facing and fibrous material.

In the future, it will be necessary to investigate the acoustical behavior of facings and films with other types of porous absorbing material used for noise barriers.

\section{REFERENCES}

[1] W. A. Davern, "Perforated facings backed with porous materials as sound absorbers - An experimental study - ," Appl. Acoust., 10, 85-112 (1977).

[2] R. H. Bolt, "On the design of perforated facings for acoustic materials," J. Acoust. Soc. Am., 19, 917-921 (1977).

[3] U. Ingard and R. H. Bolt, "Absorption characteristics of acoustic material with perforated facings," J. Acoust. Soc. Am., 23, 533-540 (1951).

[4] U. Ingard, "Perforated facing and sound absorption," $J$. Acoust. Soc. Am., 26, 151-154 (1954).

[5] K. P. Byrne, "Calculation of the specific normal impedance of perforated facing - Porous backing constructions," Appl. Acoust., 13, 43-55 (1980).

[6] P. Guignouard, M. Meisser, J. F. Allard, P. Rebillard and C. Depollier, "Prediction and measurement of the acoustical impedance and absorption coefficient at oblique incidence of porous layers with perorated facings," Noise Control Eng. J., 36, 129-135 (1991).

[7] D. Takahashi, "A new method for predicting the sound absorption of perforated absorber systems," Appl. Acoust., 51, 71-84 (1998).

[8] N. Voronina, "Acoustic properties of synthetic films," Appl. Acoust., 49, 127-140 (1996).

[9] K. Sakagami, M. Kiyama, M. Morimoto and D. Takahashi, "Sound absorption of a cavity-backed Membrane: A step towards design method for membrane-type absorbers," Appl. Acoust., 49, 237-247 (1996).

[10] J. P. Parkinson, J. R. Pearse and M. D. Latimer, "Sound absorption of elastic framed porous materials in combination with impervious films: Effect of bonding," Appl. Acoust., 63, 819-828 (2002).

[11] ISO 10534-2: 1998, "Acoustics — Determination of sound absorption coefficient and impedance in impedance tubes Part 2: Transfer-function method" (1998).

[12] H. Kuttruff, Room Acoustics, 2nd ed. (Applied Science Publisher Ltd., London, 1979), pp. 131-132.

[13] S. Sugie, J. Yoshimura and H. Ogawa, "Influence of facing for fibrous sound absorber set in noise barrier," CD-ROM Proc. Inter-noise 2002 (2002).

[14] Y. Miki, "Acoustic properties of porous materials -Modification of Delany-Bazley models_," J. Acoust. Soc. Jpn. (E), 11, 19-24 (1990). 